\title{
Transient Probability Functions: A Sample Path Approach
}

\author{
Michael L. Green ${ }^{1}$, Alan Krinik ${ }^{1}$, Carrie Mortensen ${ }^{1}$, Gerardo Rubino ${ }^{2}$, \\ and Randall J. Swift ${ }^{1}$ \\ ${ }^{1}$ Department of Mathematics, California State Polytechnic University, Pomona, CA 91768, USA, \\ \{mlgreen, ackrinik, camortensen, rjswift\}ecsupomona.edu \\ ${ }^{2}$ IRISA/INRIA, Campus Universitaire de Beaulieu, 35042 Rennes Cedex, France, rubino@irisa.fr
}

\begin{abstract}
A new approach is used to determine the transient probability functions of Markov processes. This new solution method is a sample path counting approach and uses dual processes and randomization. The approach is illustrated by determining transient probability functions for a three-state Markov process. This approach also provides a way to calculate transient probability functions for Markov processes which have specific sample path characteristics.
\end{abstract}

Keywords: sample paths; dual processes; transient probability functions; Markov process; randomization. Mathematics Subject Classifications: 60K25, 60J27.

\section{Introduction}

In this paper, we discuss how randomization and dual processes may be employed to determine transient probability functions of Markov processes. Our approach is based upon counting sample paths. Randomization (also known as uniformization) became popular in the 1950's and 1960's as a numerical method for approximating transient probability functions of intractable queueing systems, see [GH85], [Gra91]. In the 1990's, randomization and lattice path combinatorics were used to obtain analytic solutions of birth-death processes, see [BKM97], [KMKC97], [LPRS93]. Meanwhile, the theory of dual processes also emerged in the 1950's through the pioneering work of Karlin and McGregor and continued to be developed throughout the 1970's, see sections 7.4 and 8.2 of [And91] for a nice summary of duality results. Recently, dual processes have been used, along with randomization and path counting, to simplify the calculation of transient probability functions of non birth-death, Markov processes, cf. [KMSC02] and $\left[\mathrm{KRM}^{+} 03\right]$. Dual processes are turning out to be a particularly useful tool in the transient analysis of Markovian systems having an infinite number of states. Standard combinatorial techniques found, for example, in [Mar98] are sufficient to count the sample paths appearing in this article. However, when more specialized results in lattice path combinatorics are needed, the classical references are [Moh79] and [Nar79].

To illustrate the effectiveness of randomization and dual processes in our sample path counting approach, we require a suitable Markov process to use as an example to demonstrate the methodology. We 
have chosen a simple, three-state Markov process, having transitions diagrammed in Figure 1, as a vehicle to illustrate the sample path counting approach. There are several reasons for this selection. For the three-state Markov process, the ideas behind this new approach are clear and easy to present. A three state space is an appropriate setting to introduce the notion of a dual process and first observe how the analysis simplifies on the dual transition diagram as opposed to the equations required for the original process. In this article, the analysis on the dual diagram leads to roots of a quadratic whereas the original process diagram requires the roots of a cubic equation. More generally, however, the major advantage of dual processes to the solution approach is to replace path counting on a recurrent Markov chain by sample path counting on an absorbing Markov chain, see [KMSC02] and [ $\mathrm{KRM}^{+} 03$ ]. In addition to finding the usual transition probability functions, the three-state Markov process also provides an accessible example to display the path counting approach to calculate transient probabilities having pre-specified sample path conditions. As an illustration, we determine the probability of going from state 1 to state 2 in time $t$ without hitting state 0 anytime along the way in the three-state Markov process. This method generalizes to different Markov processes and provides a technique for future researchers to calculate time-dependent transitional probability functions having specific sample path restrictions.

\section{Transient Probability Functions of a Three-state Markov Pro- cess}

Consider a three-state Markov process having transition rate diagram, Figure 1.

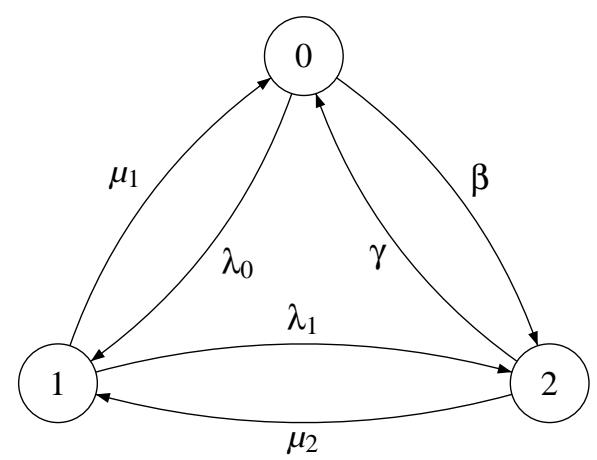

Fig. 1: A generic 3-state Markov process

where $\lambda_{0}, \lambda_{1}, \mu_{1}, \mu_{2}, \beta$ and $\gamma$ are all nonnegative constant rates having $Q$-matrix

$$
Q=\left[\begin{array}{ccc}
-\left(\lambda_{0}+\beta\right) & \lambda_{0} & \beta \\
\mu_{1} & -\left(\lambda_{1}+\mu_{1}\right) & \lambda_{1} \\
\gamma & \mu_{2} & -\left(\mu_{2}+\gamma\right)
\end{array}\right]
$$

This Markov process includes several important special cases. For example, if $\beta=\gamma=0$, we have the general three-state birth-death process which itself includes as special cases common queueing systems $\mathrm{M} / \mathrm{M} / 1 / 2$ and $\mathrm{M} / \mathrm{M} / 2 / 2$. However, to treat the general case when all transitions are possible, we restrict 
ourselves to assuming that $\lambda_{0}, \lambda_{1}, \mu_{1}, \mu_{2}, \beta$ and $\gamma$ are all positive quantities. The analysis is similar (and simpler) if some of these transition rates are zero and therefore missing from the diagram in Figure 1.

The dual process of the process described in Figure 1 is well defined (see section 7.4 of [And91]), if and only if $\mu_{1} \geq \gamma$ and $\lambda_{1} \geq \beta$ and has transition rate diagram Figure 2 .

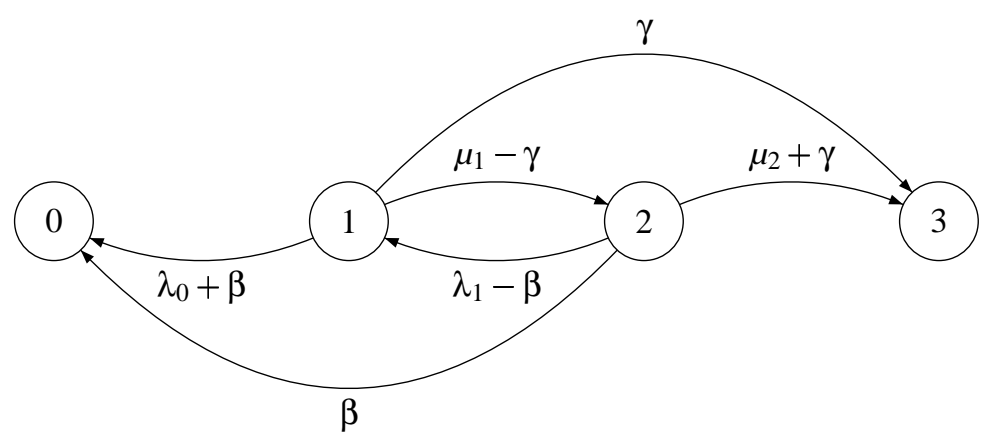

Fig. 2: The dual of the process of Figure 1

Again, to consider the case when all pictured transitions occur we suppose that $\mu_{1}>\gamma$ and $\lambda_{1}>\beta$. The $Q$-matrix of this dual process in Figure 2 is

$$
Q^{*}=\left[\begin{array}{cccc}
0 & 0 & 0 & 0 \\
\lambda_{0}+\beta & -\left(\lambda_{0}+\mu_{1}+\beta\right) & \mu_{1}-\gamma & \gamma \\
\beta & \lambda_{1}-\beta & -\left(\lambda_{1}+\mu_{2}+\gamma\right) & \mu_{2}+\gamma \\
0 & 0 & 0 & 0
\end{array}\right]
$$

If we call the transient probability functions of the processes shown in Figures 1 and $2, P_{i, j}(t)$ and $P_{i, j}^{*}(t)$ respectively, then from duality theory (see [And91]) we have

$$
P_{i, j}(t)=\sum_{k=0}^{i}\left[P_{j, k}^{*}(t)-P_{j+1, k}^{*}(t)\right] \quad \text { for } i, j=0,1,2
$$

For simplicity in presentation, we restrict ourselves to $i=0$, so

$$
P_{0, j}(t)=P_{j, 0}^{*}(t)-P_{j+1,0}^{*}(t) \quad \text { for } j=0,1,2
$$

The dual approach determines $P_{0, j}(t)$ by finding $P_{j, 0}^{*}(t)$ through equation (1).

One essential difference between our original three-state Markov process of Figure 1 and its dual pictured in Figure 2 is that the dual process has two absorbing states. It turns out (as we will see) that having absorbing states makes it easier to determine transient probability functions of the dual process as compared to the original three state Markov process of Figure 1. Rewriting equation (1) we obtain the three 
relations

$$
\begin{aligned}
& P_{0,0}(t)=1-P_{1,0}^{*}(t) \\
& P_{0,1}(t)=P_{1,0}^{*}(t)-P_{2,0}^{*}(t) \\
& P_{0,2}(t)=P_{2,0}^{*}(t)
\end{aligned}
$$

This completes the duality part of our sample path counting approach.

On a finite state space, say $\{0,1, \ldots, n-1\}$, the transient probability functions $P_{i, j}(t)$, of a Markov process are always determined by solving the Kolmogorov backward or forward equations (see for instance [BW90] or [GH85]). The result is a system of differential equations that may be written in matrix form as

$$
P^{\prime}(t)=Q P(t)=P(t) Q,
$$

where $P(t)=\left(P_{i, j}(t)\right)_{i, j \in\{0,1, \ldots, n-1\}}$ and $Q=\left(q_{i, j}\right)_{i, j \in\{0,1, \ldots, n-1\}}$ and $Q$ is the transition is the transition rate matrix of the process. The solution to the Kolmogorov differential equation can then be written

$$
P(t)=e^{Q t} .
$$

For a general Markov process on a finite state space $\{0,1, \ldots, n-1\}$, consider a Markov chain called the associated randomized chain having one-step transition probabilities defined by:

$$
\begin{gathered}
p_{i, j}=\frac{q_{i, j}}{m}, \quad i \neq j, \\
p_{i, i}=1+\frac{q_{i, i}}{m},
\end{gathered}
$$

where $m$ is chosen such that $\max _{i}\left|q_{i, i}\right| \leq m<\infty$. The following theorem, called randomization (or uniformization), is a well-known result used primarily for the numerical computation of the transition probability functions of a Markov process [GH85], [Gra91]. It is stated here for a Markov process on a finite state space but it applies, more generally, for any Markov process on countable state space, having uniformly bounded diagonal transition rates in the $Q$-matrix (see for instance [And91]).

Theorem 2.1 Suppose a Markov process on $\{0,1, \ldots, n-1\}$ has transition rate matrix $Q$ with $\max _{i}\left|q_{i, i}\right| \leq$ $m<\infty$. Then the transition probability functions $P_{i, j}(t)$ may be written as

$$
P_{i, j}(t)=\sum_{n=0}^{\infty} e^{-m t} \frac{(m t)^{n}}{n !} P_{i, j}^{(n)}
$$

where $P_{i, j}^{(n)}$ is the $n$-step transition probability of the associated randomized Markov chain.

It should be noted that $P_{i, j}(t)$ is completely determined once $P_{i, j}^{(n)}$ is "known". For examples of solving for $P_{i, j}(t)$ by finding $P_{i, j}^{(n)}$, see [BKM97], [KMKC97], [KMSC02], [KRM ${ }^{+}$03], [LPRS93].

Applying randomization to the dual process in Figure 2, the following associated Markov chain is obtained when we assume, without loss of generality, that $m=\lambda_{1}+\mu_{2}+\gamma>\lambda_{0}+\mu_{1}+\beta$ :

where

$$
p_{0}=\frac{\lambda_{0}+\beta}{m}, \quad p_{1}=\frac{\lambda_{1}-\beta}{m}, \quad q_{1}=\frac{\mu_{1}-\gamma}{m}, \quad q_{2}=\frac{\mu_{2}+\gamma}{m},
$$




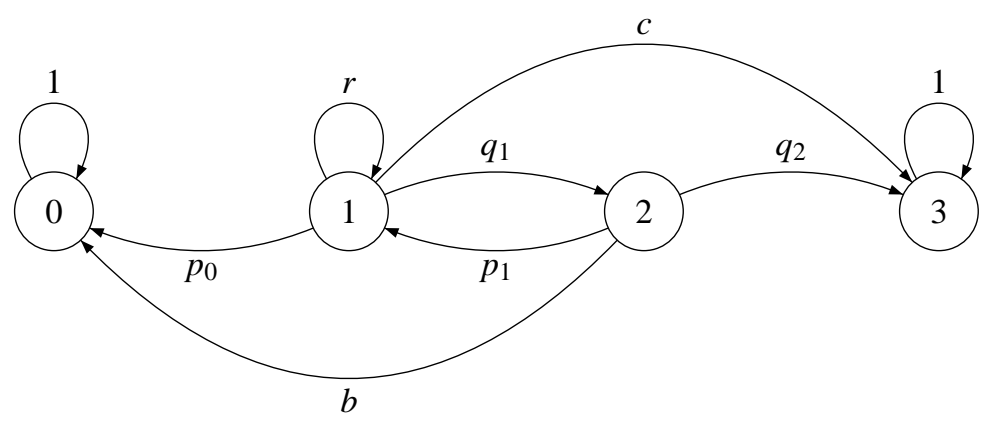

Fig. 3: The randomization of the process depicted in Figure 2

$$
b=\frac{\beta}{m}, \quad c=\frac{\gamma}{m}, \quad r=1-\left(c+p_{0}+q_{1}\right) .
$$

It follows from earlier assumptions that all of these transition probabilities are nonzero. Note that if we assumed $m=\lambda_{0}+\mu_{1}+\beta>\lambda_{1}+\mu_{2}+\gamma$, then the loop would appear on state 2 instead of state 1 in Figure 3 . If $m=\lambda_{0}+\mu_{1}+\beta=\lambda_{1}+\mu_{2}+\gamma$, there would be no loop at either state 1 or 2 . Randomization gives us

$$
P_{j, 0}^{*}(t)=\sum_{n=0}^{\infty} e^{-m t} \frac{(m t)^{n}}{n !} P_{j, 0}^{*(n)}, \quad \text { for } j=0,1,2,3,
$$

where $P_{j, 0}^{*(n)}$ is the $n$-step transition probability of going from state $j$ to state 0 in Figure 3 . We now find $P_{j, 0}^{*(n)}$ by a one-step backward analysis. This will lead to $P_{j, 0}^{*}(t)$, for $j=0,1,2,3$ which in turn gives us $P_{0, j}(t)$ by equations (2). On Figure 3,

$$
P_{1,1}^{*(n+1)}=r P_{1,1}^{*(n)}+p_{1} P_{1,2}^{*(n)} \quad \text { and } \quad P_{1,2}^{*(n+1)}=q_{1} P_{1,1}^{*(n)} .
$$

So,

$$
P_{1,1}^{*(n+1)}=r P_{1,1}^{*(n)}+p_{1} q_{1} P_{1,1}^{*(n-1)}
$$

which is a constant coefficient, second order, linear recurrence relation. Therefore, by the theory of linear, constant coefficient recurrences, see [Mar98],

$$
P_{1,1}^{*(n)}=A r_{1}^{n}+B r_{2}^{n}, \quad \text { for } n \geq 0,
$$

where $A$ and $B$ are constants determined by the initial values of the recurrence and $r_{1}, r_{2}$ are roots of the quadratic $x^{2}-r x-p_{1} q_{1}=0$, that is,

$$
r_{1}, r_{2}=\frac{r \pm \sqrt{r^{2}+4 p_{1} q_{1}}}{2} .
$$

Here,

$$
A=\frac{r-r_{2}}{r_{1}-r_{2}} \quad \text { and } \quad B=\frac{r_{1}-r}{r_{1}-r_{2}} .
$$


Note that $r_{1}, r_{2} \neq 1$. Similarly, by combining

$$
P_{1,0}^{*(n+1)}=P_{1,0}^{*(n)}+p_{0} P_{1,1}^{*(n)}+b P_{1,2}^{*(n)},
$$

with the preceding recurrence relations yields

$$
P_{1,0}^{*(n+1)}=P_{1,0}^{*(n)}+p_{0} P_{1,1}^{*(n)}+b q_{1} P_{1,1}^{*(n-1)} .
$$

After re-indexing, this equation may be written as

$$
P_{1,0}^{*(k+1)}-P_{1,0}^{*(k)}=p_{0} P_{1,1}^{*(k)}+b q_{1} P_{1,1}^{*(k-1)} .
$$

Summing over $k$ from 1 to $n-1$ gives

$$
\begin{aligned}
P_{1,0}^{*(n)}-P_{1,0}^{*(1)} & =\sum_{k=1}^{n-1}\left[p_{0} P_{1,1}^{*(k)}+b q_{1} P_{1,1}^{*(k-1)}\right] \\
& =\sum_{k=1}^{n-1}\left[p_{0}\left(A r_{1}^{k}+B r_{2}^{k}\right)+b q_{1}\left(A r_{1}^{k-1}+B r_{2}^{k-1}\right)\right]
\end{aligned}
$$

This gives

$$
\begin{aligned}
P_{1,0}^{*(n)}= & p_{0}+\sum_{k=1}^{n-1}\left[A\left(p_{0}+\frac{b q_{1}}{r_{1}}\right) r_{1}^{k}+B\left(p_{0}+\frac{b q_{1}}{r_{2}}\right) r_{2}^{k}\right] \\
= & p_{0}+A\left(p_{0}+\frac{b q_{1}}{r_{1}}\right) \frac{1-r_{1}^{n}}{1-r_{1}}+B\left(p_{0}+\frac{b q_{1}}{r_{2}}\right) \frac{1-r_{2}^{n}}{1-r_{2}} \quad\left(\text { since } r_{1}, r_{2} \neq 1\right) \\
= & {\left[p_{0}+\frac{A\left(p_{0}+b q_{1} r_{1}^{-1}\right)}{1-r_{1}}+\frac{B\left(p_{0}+b q_{1} r_{2}^{-1}\right)}{1-r_{2}}\right] } \\
& -\left[\frac{A\left(p_{0}+b q_{1} r_{1}^{-1}\right)}{1-r_{1}}\right] r_{1}^{n}-\left[\frac{B\left(p_{0}+b q_{1} r_{2}^{-1}\right)}{1-r_{2}}\right] r_{2}^{n} \quad(\text { for } n \geq 2) .
\end{aligned}
$$

Note that $P_{1,0}^{*(0)}=0$ and $P_{1,0}^{*(1)}=p_{0}$. Let

$$
\begin{gathered}
C_{0}=p_{0}+\frac{A\left(p_{0}+b q_{1} r_{1}^{-1}\right)}{1-r_{1}}+\frac{B\left(p_{0}+b q_{1} r_{2}^{-1}\right)}{1-r_{2}}, \\
C_{1}=-\frac{A\left(p_{0}+b q_{1} r_{1}^{-1}\right)}{1-r_{1}} \text { and } C_{2}=-\frac{B\left(p_{0}+b q_{1} r_{2}^{-1}\right)}{1-r_{2}} .
\end{gathered}
$$

Note that $C_{0}=p_{0}-C_{1}-C_{2}$. Substituting these results into the randomization formula and using the 
Taylor series for the exponential function produces

$$
\begin{aligned}
P_{1,0}^{*}(t)= & \sum_{n=0}^{\infty} e^{-m t} \frac{(m t)^{n}}{n !} P_{1,0}^{*(n)} \\
= & e^{-m t} m t p_{0}+\sum_{n=2}^{\infty} e^{-m t} \frac{(m t)^{n}}{n !}\left[C_{0}+C_{1} r_{1}^{n}+C_{2} r_{2}^{n}\right] \\
= & e^{-m t} m t p_{0} \\
& +e^{-m t}\left[C_{0}\left(e^{m t}-1-m t\right)+C_{1}\left(e^{m r_{1} t}-1-m r_{1} t\right)+C_{2}\left(e^{m r_{2} t}-1-m r_{2} t\right)\right] \\
= & C_{0}+\left[m t p_{0}-\left(C_{0}+C_{1}+C_{2}\right)-\left(C_{0} m t+C_{1} m r_{1} t+C_{2} m r_{2} t\right)\right] e^{-m t} \\
\quad & +C_{1} e^{m\left(r_{1}-1\right) t}+C_{2} e^{m\left(r_{2}-1\right) t} \\
= & C_{0}-p_{0} e^{-m t}-\left[C_{0}-p_{0}+C_{1} r_{1}+C_{2} r_{2}\right] m t e^{-m t}+C_{1} e^{m\left(r_{1}-1\right) t}+C_{2} e^{m\left(r_{2}-1\right) t} .
\end{aligned}
$$

In a similar way, $P_{2,0}^{*}(t)$ can be found by using recurrence relations to find $P_{2,0}^{*(n)}$. From Figure 3 , it follows that

$$
P_{2,0}^{*(n+1)}=P_{2,0}^{*(n)}+p_{0} P_{2,1}^{*(n)}+b P_{2,2}^{*(n)}, \quad P_{2,1}^{*(n+1)}=r P_{2,1}^{*(n)}+p_{1} P_{2,2}^{*(n)}, \quad P_{2,2}^{*(n+1)}=q_{1} P_{2,1}^{*(n)} .
$$

So,

$$
P_{2,1}^{*(n+1)}=r P_{2,1}^{*(n)}+p_{1} q_{1} P_{2,1}^{*(n-1)},
$$

is the same constant coefficient, second order, linear recurrence relation found earlier. So, as before

$$
P_{2,1}^{*(n)}=C r_{1}^{n}+D r_{2}^{n}
$$

for $n \geq 0$, where $C$ and $D$ are constants determined by the initial values of the recurrence, and $r_{1}, r_{2}$ are given in (3). Noting that $P_{2,1}^{*(0)}=0$ and $P_{2,1}^{*(1)}=p_{1}$ leads this time to

$$
C=\frac{p_{1}}{r_{1}-r_{2}} \quad \text { and } \quad D=\frac{p_{1}}{r_{2}-r_{1}}=-C \text {. }
$$

Substituting this expression for $P_{2,1}^{*(n)}$ into $P_{2,0}^{*(n+1)}=P_{2,0}^{*(n)}+p_{0} P_{2,1}^{*(n)}+b q_{1} P_{2,1}^{*(n-1)}$ gives

$$
\begin{aligned}
P_{2,0}^{*(k+1)}-P_{2,0}^{*(k)} & =p_{0} P_{2,1}^{*(k)}+b q_{1} P_{2,1}^{*(k-1)} \\
& =p_{0}\left(C r_{1}^{k}+D r_{2}^{k}\right)+b q_{1}\left(C r_{1}^{k-1}+D r_{2}^{k-1}\right) \\
& =p_{0} C\left(r_{1}^{k}-r_{2}^{k}\right)+b q_{1} C\left(r_{1}^{k-1}-r_{2}^{k-1}\right) .
\end{aligned}
$$

Summing,

$$
\begin{aligned}
P_{2,0}^{*(n)}-P_{2,0}^{*(1)} & =\sum_{k=1}^{n-1}\left[p_{0} P_{2,1}^{*(k)}+b q_{1} P_{2,1}^{*(k-1)}\right] \\
& =\sum_{k=1}^{n-1}\left[p_{0}\left(C r_{1}^{k}+D r_{2}^{k}\right)+b q_{1}\left(C r_{1}^{k-1}+D r_{2}^{k-1}\right)\right] \\
& =C \sum_{k=1}^{n-1}\left[p_{0}\left(r_{1}^{k}-r_{2}^{k}\right)+b q_{1}\left(r_{1}^{k-1}-r_{2}^{k-1}\right)\right] .
\end{aligned}
$$


Note that $P_{2,0}^{*(1)}=b$. This produces

$$
P_{2,0}^{*(n)}=b+C \sum_{k=1}^{n-1}\left[\left(p_{0}+\frac{b q_{1}}{r_{1}}\right) r_{1}^{k}-\left(p_{0}+\frac{b q_{1}}{r_{2}}\right) r_{2}^{k}\right] .
$$

From here, it is seen that the analysis goes forward precisely as in the preceding calculation for $P_{1,0}^{*(n)}$ with $A$ and $B$ being replaced by $C$ and $D(=-C)$. Let

$$
\begin{gathered}
D_{0}=b+\frac{C\left(p_{0}+b q_{1} r_{1}^{-1}\right)}{1-r_{1}}+\frac{D\left(p_{0}+b q_{1} r_{2}^{-1}\right)}{1-r_{2}}, \\
D_{1}=-\frac{C\left(p_{0}+b q_{1} r_{1}^{-1}\right)}{1-r_{1}} \text { and } D_{2}=-\frac{D\left(p_{0}+b q_{1} r_{2}^{-1}\right)}{1-r_{2}} .
\end{gathered}
$$

Then substituting these results into the randomization formula and using the Taylor series for the exponential function together with $P_{2,0}^{*(0)}=0$ and $P_{2,0}^{*(1)}=b$ produces

$$
P_{2,0}^{*}(t)=D_{0}-b e^{-m t}-\left[D_{0}-b+D_{1} r_{1}+D_{2} r_{2}\right] m t e^{-m t}+D_{1} e^{m\left(r_{1}-1\right) t}+D_{2} e^{m\left(r_{2}-1\right) t} .
$$

This expression and the previously determined expression,

$$
P_{1,0}^{*}(t)=C_{0}-p_{0} e^{-m t}-\left[C_{0}-p_{0}+C_{1} r_{1}+C_{2} r_{2}\right] m t e^{-m t}+C_{1} e^{m\left(r_{1}-1\right) t}+C_{2} e^{m\left(r_{2}-1\right) t}
$$

may be substituted back into (2) to give the explicit transient probability functions for the three-state Markov process. Note: for $i, j=0,1,2, P_{i, j}(t)$ may also be found in the same manner.

\section{A non standard transient probability calculation}

We again consider the three-state Markov process having transition rate diagram, Figure 1. This time, we assume $m=\lambda_{0}+\beta, m>\lambda_{1}+\mu_{1}$ and $m>\mu_{2}+\gamma$. Randomizing the process described in Figure 1 using these assumptions, gives the following Markov chain, diagrammed in Figure 4, where

$$
\begin{gathered}
p_{0}=\frac{\lambda_{0}}{m}, \quad p_{1}=\frac{\lambda_{1}}{m}, \quad q_{1}=\frac{\mu_{1}}{m}, \quad q_{2}=\frac{\mu_{2}}{m}, \\
b=\frac{\beta}{m}, \quad c=\frac{\gamma}{m}, \quad r_{1}=1-\left(p_{1}+q_{1}\right), \quad r_{2}=1-\left(c+q_{2}\right) .
\end{gathered}
$$

Note: these quantities are defined differently here than in Section 2.

We wish to calculate the transient probability function $\widetilde{P}_{1,2}(t)$, which is defined to be the probability of starting at state 1 and ending at state 2 in Figure 1, after some time $t$, but never reaching state 0 along the way. Using the one-step backward analysis as before, we have

$$
\widetilde{P}_{1,2}^{(n+1)}=p_{1} \widetilde{P}_{1,1}^{(n)}+r_{2} \widetilde{P}_{1,2}^{(n)}, \quad \widetilde{P}_{1,1}^{(n+1)}=r_{1} \widetilde{P}_{1,1}^{(n)}+q_{2} \widetilde{P}_{1,2}^{(n)}
$$

So,

$$
\widetilde{P}_{1,2}^{(n+2)}-\left(r_{1}+r_{2}\right) \widetilde{P}_{1,2}^{(n+1)}+\left(r_{1} r_{2}-p_{1} q_{2}\right) \widetilde{P}_{1,2}^{(n)}=0 .
$$




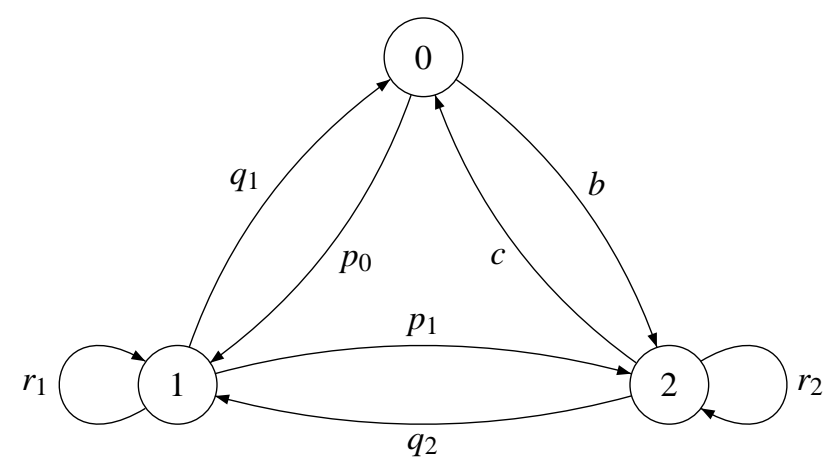

Fig. 4: The randomization of the particular case analyzed in this section

By the theory of linear constant coefficient recurrence relations, $\widetilde{P}_{1,1}^{(n)}=E \rho_{1}^{n}+F \rho_{2}^{n}$, where $E$ and $F$ are determined by the initial values of the recurrence and $\rho_{1}, \rho_{2}$ are the distinct roots of the characteristic equation of this recurrence relation, $x^{2}-\left(r_{1}+r_{2}\right) x+r_{1} r_{2}-p_{1} q_{2}=0$, which are

$$
\rho_{1}, \rho_{2}=\frac{r_{1}+r_{2} \pm \sqrt{\left(r_{1}-r_{2}\right)^{2}+4 p_{1} q_{2}}}{2}
$$

It works out that

$$
E=\frac{p_{1}}{\sqrt{\left(r_{1}-r_{2}\right)^{2}+4 p_{1} q_{2}}}
$$

and $F=-E$. Thus, $\widetilde{P}_{1,2}^{(n)}=E\left(\rho_{1}^{n}-\rho_{2}^{n}\right)$ for $n \geq 2$. Substituting this into the randomization formula and using the Taylor series for the exponential function along with $\widetilde{P}_{1,2}^{(0)}=0$ and $\widetilde{P}_{1,2}^{(1)}=p_{1}$ produces

$$
\widetilde{P}_{1,2}(t)=p_{1} e^{-m t}+E\left[e^{m\left(\rho_{1}-1\right) t}-e^{m\left(\rho_{2}-1\right) t}\right]+E m\left(\rho_{2}-\rho_{1}\right) t e^{-m t}
$$

\section{Remarks}

1. $\widetilde{P}_{1,1}(t)$ may be found using the same method. In queueing theory, $\widetilde{P}_{1,1}(t)+\widetilde{P}_{1,2}(t)$ is the probability of starting with one customer in the system and being busy throughout the time interval $[0, t]$. This is called the busy period distribution, see [GH85].

2. The reader is invited to generalize the example within Section 3 to determine the probability of traveling from state 1 to state 2 in time $t$ hitting the 0 state exactly once along the way for the three-state Markov process of Figure 1.

3. In Section 2 on solving the transient probability functions, in order for the dual process to have positive off-diagonal elements we assumed $\mu_{1}>\gamma$ and $\lambda_{1}>\beta$. Alternatively, it suffices to have $\mu_{1}<\gamma$ and $\mu_{2} \geq \lambda_{0}$ or $\lambda_{1}<\beta$ and $\mu_{2} \leq \lambda_{0}$. These alternative conditions emerge from re-labeling the states and insisting that all off-diagonal elements be positive for the newly shuffled $Q$-matrix. For the sake of generality, it is desirable to relax as many $Q$-matrix prerequisites as possible. 


\section{References}

[And91] W.J. Anderson. Continuous-Time Markov Chains. An Applications-Oriented Approach. Springer-Verlag, New York, 1991.

[BKM97] W. Bhm, A. Krinik, and S.G. Mohanty. The combinatorics of birth-death processes and applications to queues. Queueing Systems, 26:255-267, 1997.

[BW90] R.N. Bhattacharya and E.C. Waymire. Stochastic Processes With Applications. John Wiley \& Sons, New York, 1990.

[GH85] D. Gross and C.M. Harris. Fundamentals of Queueing Theory (Second Edition). John Wiley \& Sons, New York, 1985.

[Gra91] W.K. Grassmann. Finding transient solutions in markovian event systems through randomization. In W.J. Stewart, editor, Numerical Solution of Markov Chains, New York, 1991. Marcel Dekker.

[KMKC97] A. Krinik, D. Marcus, D. Kalman, and T. Cheng. Transient solution of the M/M/1 queueing system via randomization. In J. Goldstein, N. Gretsky, and J.J. Uhl, editors, Stochastic Processes and Functional Analysis, volume 186, pages 137-145. Marcel Dekker, New York, Lecture Notes in Pure and Applied Mathematical Series edition, 1997.

[KMSC02] A. Krinik, D. Marcus, R. Shiflett, and L. Chu. Transient probabilities of a single server priority queueing system. Journal of Statistical Planning and Inference, 101:185-190, 2002.

$\left[\mathrm{KRM}^{+}\right.$03] A. Krinik, G. Rubino, D. Marcus, R.J. Swift, H. Kasfy, and H. Lam. Dual processes to solve single server systems. To appear in Journal of Statistical Planning and Inference, 2003.

[LPRS93] P. Leguesdron, J. Pellaumail, G. Rubino, and B. Sericola. Transient solution of the M/M/1 queue. Adv. Appl. Probability, 25:702-713, 1993.

[Mar98] D. Marcus. Combinatorics. A Problem Oriented Approach. The Mathematical Association of America, Washington, 1998.

[Moh79] S.G. Mohanty. Lattice Path Counting and Applications. Academic Press, New York, 1979.

[Nar79] T.V. Narayana. Lattice Path Combinatorics With Statistical Applications. University of Toronto Press, Toronto, 1979. 